\title{
Les carcinomes de primitif inconnu de la tête et du cou
}

\section{T. Kennel $^{1}$, R. Garrel ${ }^{1 *}$, V. Costes $^{2}$, P. Boisselier ${ }^{3}$, L. Crampette ${ }^{1}$, V. Favier ${ }^{1}$}

${ }^{1}$ Département d'ORL et Chirurgie Cervico Faciale, CHRU de Montpellier, Hôpital Universitaire Gui de Chauliac, Université de Montpellier, 80, avenue Augustin-Fliche, 34295 Montpellier cedex 5, France

${ }^{2}$ Département de biopathologie cellulaire et tissulaire des tumeurs, équipe médicale anatomie et cytologie pathologiques, CHRU de Montpellier, Hôpital Universitaire Gui-de-Chauliac, Université de Montpellier, 80, avenue Augustin-Fliche, 34295 Montpellier cedex 5, France.

${ }^{3}$ Département de radiothérapie, Institut du Cancer de Montpellier, 208, avenue des Apothicaires, 34298 Montpellier, France.

*auteur correspondant

Adresse e-mail : r-garrel@ chu-montpellier.fr (Renaud GARREL)

CHU de Montpellier, Hôpital Gui de Chauliac

80 avenue Augustin Fliche

34295 Montpellier cedex 5

Tél: 0467336825

Fax: 0467336728 


\section{Résumé :}

En pathologie cervico faciale de l'adulte, les carcinomes de primitif inconnu sont définis par la présence de métastase(s) ganglionnaire(s) dont le point de départ anatomique est inconnu lors de la prise en charge thérapeutique initiale. Ils représentent jusqu'à $5 \%$ des cancers de la tête et du cou. Leurs modes de présentation peuvent évoquer une pathologie bénigne, occasionnant alors un retard et des incohérences dans la prise en charge carcinologique. La stratégie diagnostique devant une adénopathie prévalente suspecte de néoplasie nécessite la réalisation d'un bilan exhaustif permettant d'une part de diagnostiquer la malignité de la masse et d'autre part, d'identifier le site du primitif dans $45 \%$ à $80 \%$ des cas selon les séries. Les différents types histologiques rencontrés sont le carcinome épidermoïde, les carcinomes thyroïdiens, les adénocarcinomes, les carcinomes neuroendocrines et les carcinomes indifférenciés. Une association au papillomavirus humain et à l'Epstein Barr virus est parfois retrouvée et oriente alors la prise en charge. Les objectifs de cet article sont d'apporter au clinicien les outils nécessaires dans la démarche diagnostique à partir des connaissances actuelles en clinique, imagerie et anatomopathologie, ainsi que détailler les options thérapeutiques.

Mots clés : Métastases d'origine inconnue, tumeurs oto-rhino-laryngologiques, métastase lymphatique 


\section{Introduction}

Un cancer métastatique de site primitif inconnu est défini par la présence de métastase(s) dont le point de départ anatomique est inconnu au moment de la prise en charge thérapeutique initiale [1]. Dans la région de la tête et du cou, ces métastases concernent les structures ganglionnaires et représentent 1,5 à $5 \%$ de l'ensemble des tumeurs [2,3]. Une conduite diagnostique rigoureuse est indispensable avant d'établir le diagnostic de cancer de primitif inconnu (CAPI) des voies aérodigestives supérieures (VADS). Lorsque le bilan réussit à identifier le site primitif, le terme de métastase ganglionnaire sans primitif retrouvé est alors inapproprié. Un CAPI de la tête et du cou représente une difficulté diagnostique, du fait de son mode présentation inhabituel, source de retard de prise en charge [4,5], mais également peut être source d'incohérences voire d'erreurs thérapeutiques.

Cet article a pour objectifs de détailler les données de la littérature concernant les types histologiques, la stratégie diagnostique à adopter, et les options thérapeutiques devant un CAPI cervical.

\section{Discussion}

\subsection{Histopathologie des CAPI cervicaux}

\section{Carcinomes épidermoïdes : généralités}

Les carcinomes épidermoïdes représentent $53 \%$ à $77 \%$ des CAPI cervicaux. La forme la plus fréquente est le carcinome épidermoïde commun retrouvé dans $85 \%$ des cas. Les $15 \%$ restant concernent des variants (verruqueux, papillaire, à cellules fusiformes, adénosquameux, indifférenciés et basaloïdes) [4].

Il semble exister un lien entre papillomavirus humain (HPV) et CAPI. Les séries nord-Américaines retrouvent jusqu'à $80 \%$ des primitifs dans l'oropharynx au terme d'un bilan diagnostique incluant la chirurgie robotique, avec une association HPV et CAPI épidermoïde pouvant atteindre jusqu'à 90\% [6-8] . Cependant, les résultats des différentes études publiées ou à venir que ce soit sur le taux d'identification du primitif ou les notions de désescalade thérapeutique doivent être interprétés avec prudence en raison de la variabilité géographique de la prévalence de l'HPV en ORL. L'étude Papillophar, réalisée entre 2009 et 2012 sur 14 centres hospitaliers français, retrouvait seulement $27 \%$ d'association à l'HPV dans les cancers de l'oropharynx, ce qui est nettement inférieur aux données d'Amérique du Nord[9]. Par ailleurs, la prévalence en France de l'HPV dans les CAPI épidermoïdes n'est pas connue.

Concernant l'expression de cancers de l'oropharynx sous forme de CAPI cervical, une explication est donnée par la structure histologique du tissu amygdalien qui est composé de tissu lymphoïde et épithélial [10]. 
L'épithélium de surface en continuité avec la muqueuse de l'oropharynx est parsemé de cryptes qui interviennent dans la fonction immunitaire de l'amygdale. Au cours des infections par HPV de haut risque oncogène, le virus infectant les cellules basales des épithéliums malpighiens favorise le développement tumoral au fond des cryptes amygdaliennes. L'épithélium des cryptes amygdaliennes repose sur une membrane basale discontinue et bordée de vaisseaux sanguins intraépithéliaux, permettant à des tumeurs millimétriques de métastaser sans avoir acquis les altérations génétiques nécessaires à l'invasion stromale (ainsi le terme de « carcinome in situ » ne doit pas être utilisé pour des tumeurs localisées dans les amygdales palatines ou linguales) [11]. Ce tissu métastatique peu différencié migre précocement dans le système lymphatique, s'accompagnant souvent de remaniements nécrotiques entrainant une kystisation des métastases (figure 1).

\section{Les carcinomes épidermö̈des du nasopharynx}

On distingue les carcinomes épidermoïdes kératinisants, les carcinomes épidermoïdes non kératinisants et les carcinomes épidermoïdes basaloïdes. La forme non kératinisante est divisée en sous-groupes différencié et indifférencié et est pratiquement toujours associée au virus d'Epstein Barr (EBV) [12].

Un carcinome épidermoïde associé à l'EBV est donc à considérer comme un primitif nasopharyngé occulte [13].

\section{Quels sont les marqueurs à rechercher devant un CAPI de type épidermoüde?}

Une recherche d'association à l'HPV et à l'EBV doit être réalisée dans le bilan d'un CAPI depuis la $8^{\mathrm{e}}$ mise à jour de classification Tumor Node Metastasis (TNM) de l'American Joint Committee on Cancer (AJCC) de 2017 (Tableaux 1,2,3) [13]. Ceci permet de classer un primitif inconnu associé à l'HPV comme originaire de l'oropharynx, et celui associé à l'EBV comme originaire du nasopharynx afin d'orienter la prise en charge thérapeutique, notamment pour la sélection des volumes cibles muqueux en radiothérapie.

L'algorithme présenté dans la Figure 2 indique dans quelles situations les tests doivent être réalisés à partir des recommandations du collège nord-Américain de pathologie (Figure 2) [14].

Sur le matériel de cytoponction ganglionnaire, pour affirmer le diagnostic de métastase kystique de carcinome épidermoïde HPV induit, il est possible d'utiliser l'une des techniques de biologie moléculaire suivante : détection de l'acide désoxyribonucléique (ADN) des HPV de hauts risques oncogènes (HR-HPV) par réaction en chaîne par polymérase (PCR), détection de l'ADN HR HPV par hybridation in situ (HIS) et détection de l'acide ribonucléique E6/E7 (ARN E6/E7) par HIS. L'immunomarquage anti p16 n'est pas suffisant sur du matériel de cytoponction seul.

Sur du matériel d'exérèse ganglionnaire, pour prouver l'origine HPV d'une métastase située dans les secteurs II ou III d'un carcinome épidermoïde non kératinisant, la recherche de la protéine p16 dans l'adénopathie est suffisante (expression intense et diffuse de p16 sur plus de 70\% des cellules avec un marquage nucléaire et cytoplasmique). 
S'il s'agit d'un carcinome épidermoïde kératinisant ou que l'adénopathie n'est pas située dans les secteurs II ou III, un test complémentaire spécifique de l'HPV (HIS ou PCR) doit être réalisé. Pour rechercher une association à l'EBV la détection d'EBER1 par HIS est la méthode la plus fiable.

\section{Diagnostics différentiels des CAPI épidermoïdes p16+ sans primitif oropharyngé}

Les métastases ganglionnaires cervicales de carcinomes épidermoïdes cutanés expriment p16 dans 20\% des cas, sans être associées aux HPV de hauts risques oncogènes $[15,16]$. Des carcinomes épidermoïdes associés à l'HPV ont été retrouvés dans le nasopharynx [17], ainsi que la cavité orale et le larynx [18]. Enfin, quel que soit le site primitif, une régression tumorale spontanée peut entrainer la disparition de la tumeur primaire, correspondant à un authentique T0 selon la classification TNM [19].

\section{Les carcinomes différenciés de la thyrö̈de}

Il s'agit principalement des carcinomes papillaires dont les métastases ganglionnaires sont plus fréquentes que dans le sous-type vésiculaire. Dans la série de Garrel et al. [20] sur 167 cas de microcarcinomes papillaires, 4,4\% des patients $(n=15)$ se sont présentés avec des adénopathies prévalentes d'un carcinome papillaire sans primitif retrouvé à l'imagerie. L'identification de cellules papillaires à la cytologie ganglionnaire ou un taux élevé de thyroglobuline dosée sur le liquide de rinçage de l'aiguille de cytoponction confirme le diagnostic [21,22]. Un carcinome médullaire de la thyroïde doit également être recherché, le dosage de la calcitonine sérique est utile, mais doit tenir compte des risques de faux positifs pouvant atteindre $69 \%$ selon les séries [23,24].

\section{Les carcinomes neuroendocrines}

Les critères morphologiques associés à l'expression de la chromogranine A et de la synaptophysine orientent vers un carcinome neuroendocrine [25]. La localisation la plus fréquente des carcinomes neuroendocrines dans les VADS est le larynx sus-glottique dont l'évolution peut être sous-muqueuse. Un carcinome de Merkel doit être évoqué en cas de carcinome neuroendocrine positif pour la cytokératine 20, suggérant ainsi une métastase d'un primitif cutané occulte ou de régression spontanée [26].

\section{Les adénocarcinomes des glandes salivaires}

Une métastase ganglionnaire d'un adénocarcinome située dans les niveaux I, II et III doit faire rechercher un primitif des glandes salivaires[27]. 
Les adénopathies métastatiques situées en région sus-claviculaire concernent des primitifs hors tête et cou dans pratiquement $1 \%$ des cas et sont majoritairement représentées par les adénocarcinomes. Les principaux sites par ordre de fréquence sont le sein, le poumon, le rein, le testicule et l'utérus [28]. Le profil tumoral réalisé à l'aide des cytokératines CK7 et CK20 permet d'identifier un groupe de sites primitifs potentiels, secondairement complété par une évaluation immunohistochimique [25].

\subsection{Conduite diagnostique}

\section{Évaluation clinique}

Le délai moyen entre la constatation d'une masse cervicale et le diagnostic de CAPI varie de 2 à 5 mois [4,5]. La présentation clinique habituelle est celle d'une masse cervicale qui évolue depuis 3 à 4 semaines. Dans la série de Grau et al. [29], il s'agissait de l'unique symptôme dans $94 \%$ des cas ( $\mathrm{n}=352)$. La douleur et la perte de poids n'étaient retrouvées que dans $9 \%$ et $7 \%$ des cas respectivement. Les conduites à tenir devant une adénopathie cervicale chronique et une adénopathie cervicale d'allure kystique sont données par les recommandations de la société française d'ORL publiées en 2010 et en 2018[30,31], résumée dans la figure 3. Pour le clinicien, l'objectif prioritaire est d'éliminer une pathologie néoplasique en recherchant des antécédents de carcinome cutané à potentiel métastatique (carcinome épidermoïde, mélanome et carcinome de Merkel), un primitif des VADS ou thyroïdien. Une attention particulière doit être portée sur le diagnostic de kyste de la deuxième fente branchiale (kyste amygdaloïde). Il s'agit d'une masse cervicale isolée antérolatérale avec des parois fines et un contenu liquidien épais (« chocolat au lait » à la cytoponction), sans critère de malignité à l'imagerie. Le mode de révélation peut être tardif, à l'âge adulte, au décours d'un épisode infectieux. Après 40 ans, ces kystes doivent être considérés d'origine métastatique jusqu'à preuve du contraire [32-34]. Dans l'étude de Pietarinen-Runtti et al. [35] sur 196 patients pris en charge pour des kystes de la deuxième fente considérés comme bénins radiologiquement, il s'agissait d'une métastase kystique d'un carcinome épidermoïde dans 3,1\% des cas $(n=6)$ ou d'une métastase kystique d'un carcinome papillaire thyroïdien occulte dans $0,5 \%$ des cas $(n=1)$. Une adénopathie kystique peut aussi être évocatrice d'un primitif induit par le papillomavirus localisé dans l'oropharynx (amygdales palatines et amygdales linguales) [3,36,37]. La localisation de l'adénopathie prévalente est une indication du site potentiel du primitif (figure 4)[38]. Les niveaux ganglionnaires majoritairement atteints dans les CAPI cervicaux sont par ordre de fréquence II, III, IV [29]. Des adénopathies bilatérales orientent vers une tumeur localisée sur la ligne médiane (plancher buccal, nasopharynx, base de langue, larynx). Le carcinome du nasopharynx métastase principalement dans les territoires IIb ou V tandis que le cancer thyroïdien occulte donne classiquement une atteinte du niveau IV [20]. Une atteinte du niveau IV (gauche = ganglion de Troisier) et $\mathrm{Vb}$ doit faire rechercher un primitif digestif, pulmonaire ou mammaire [39]. Cette répartition ganglionnaire peut néanmoins être source de confusion, car dans 10 à $15 \%$ des cas, les métastases ganglionnaires de la cavité buccale ou de l'oropharynx sont localisées dans le niveau III ou IV [40]. 
Le scanner cervical injecté, de la base du crâne aux clavicules, couplé à un scanner thoracique dans le cadre du bilan d'extension est l'examen d'imagerie de référence. Sa sensibilité varie de $49 \%$ à $94 \%$ et sa spécificité $78 \%$ à $98 \%$ dans l'identification d'une adénopathie maligne, et il précise la situation anatomique de(s) adénopathie(s) par rapport aux structures vasculo-nerveuses, évalue une extension extracapsulaire ou la présence d'adénopathies rétro pharyngées ou controlatérales [41]. L'IRM offre une meilleure analyse tissulaire que la tomodensitométrie pour la base de langue et les amygdales palatines qui sont les sites principaux des CAPI cervicaux $[42,43]$. Néanmoins, il n'est pas prouvé que l'IRM est plus sensible que le scanner pour identifier un cancer occulte des VADS [44].

\section{Cytologie}

Une cytoponction ganglionnaire réalisée au mieux, sous contrôle échographique est l'examen de première intention. Le risque de disséminer des cellules sur le trajet de ponction est négligeable [45]. Il s'agit d'un examen opérateur dépendant qui présente pour une équipe entrainée une sensibilité de $83 \%$ à $97 \%$ et une spécificité de $91 \%$ à $100 \%$ dans la recherche des cellules métastatiques [45-47]. Une cytoponction bénigne ne permet pas d'éliminer le diagnostic d'une adénopathie métastatique, surtout devant une présentation kystique, où le taux de faux négatif peut atteindre $42 \%$ [48]. Une cytoponction ciblée sur les parois du kyste améliore la positivité [49]. Une méthode simple pour confirmer un cancer différencié de la thyroïde est de réaliser un dosage de la thyroglobuline dans le liquide de rinçage de l'aiguille utilisée. Même en l'absence de cellules tumorales, une thyroglobuline élevée confirme une néoplasie d'origine thyroïdienne [21,22]. Une biopsie au trocart peut être discutée en cas de cytoponction négative ; le risque d'ensemencement tumoral serait très faible, évalué à $0,001 \%$ [50]. L'exérèse ganglionnaire partielle est à éviter du fait du taux accru de complications locales, de dissémination de cellules cancéreuses systémique et sur le trajet chirurgical [51]. Cependant, il a été rapporté l'absence d'effet néfaste de ces techniques lorsqu'un traitement complémentaire par chirurgie et/ou radiothérapie est réalisé dans le cadre d'une pathologie maligne [52,53].

\section{Tomographie par émission de positons (TEP)}

La réalisation d'une tomographie par émission de positon au 18-fluoro-désoxyglucose (FDG) est actuellement conseillée dans le bilan diagnostique d'une adénopathie cervicale métastatique prévalente. La TEP/TEP-TDM présente comme limite de résolution des tumeurs inférieures en taille à 8-10mm [42,54]. Le tissu lympho épithélial de l'anneau de Waldeyer et les glandes salivaires sont des sites de fixation physiologique du FDG, source de faux positifs. Une activité hypermétabolique est détectée jusqu'à 6 semaines sur le 
site opératoire après des biopsies et/ou amygdalectomie(s) [55]. La TEP couplée au scanner (TEP-TDM) offre une meilleure rentabilité diagnostique que la TEP seule et est maintenant l'examen de référence par rapport au TEP seul [2]. Pour Miller [56], la TEP-TDM permettait de détecter 29\% des tumeurs primitives lorsque le bilan d'imagerie par scanner et IRM était négatif. La combinaison d'une TEP-TDM à une panendoscopie aboutissait à un taux de détection de 45\%. Lorsque ces deux examens ne retrouvaient pas de primitif, la tumeur était retrouvée lors du suivi dans moins de 6\% des cas. Dans la méta-analyse de Rusthoven et al. [54] regroupant 16 études (302 patients) publiées entre 1994 et 2003, la détection d'une activité hypermétabolique correspondait à un faux positif dans 39,3\% des cas pour l'amygdale palatine ; $21 \%$ des cas pour la base de langue ; et $8 \%$ pour l'hypopharynx. La réalisation de cet examen a permis d'identifier des métastases ganglionnaires cervicales non connues dans $15,9 \%$ des cas ou à distance dans $11,2 \%$ des cas. La critique principale de ces études et des résultats présentés concerne la définition utilisée pour le terme de CAPI, car les critères d'inclusions variaient d'un examen physique seul à un scanner/IRM avec panendoscopie avant la réalisation de la TEP/TEP-TDM [57]. La sensibilité de la TEP/TEP-TDM est ainsi comprise entre 27 et $87,5 \%$ avec une valeur prédictive positive qui varie de 57 à $77 \%$ dans la détection du primitif [58-60].

\section{Panendoscopie, amygdalectomie (s) et biopsies}

Cette séquence est réalisée pour les CAPI de type épidermoïde. Une panendoscopie des VADS avec cavoscopie incluant le nasopharynx sous anesthésie générale est systématiquement réalisée. Il faut rechercher des lésions sous-muqueuses, parfois millimétriques, notamment en région amygdalienne et en base de langue, en s'aidant de la palpation digitale. Lorsque la panendoscopie et la TEP-TDM ne permettent pas d'orienter les biopsies, une amygdalectomie palatine homolatérale à l'adénopathie métastatique couplée à des biopsies de base de langue sont réalisées. L'objectif est d'identifier un cancer sous-muqueux ou localisé au fond des cryptes du tissu lymphoépithélial des amygdales palatines et linguales. Ces sites représentent jusqu'à $90 \%$ des sites de primitifs inconnus se déclarant ultérieurement dans le suivi du patient [61].

L'intérêt d'identifier le primitif par cette technique chirurgicale est de définir un volume cible optimum en cas d'indication de radiothérapie, de diminuer les doses d'irradiations aux autres sites muqueux des VADS et d'améliorer le suivi post thérapeutique [62,63]. Les analyses histologiques du tissu amygdalien doivent être réalisées à partir de coupes fines tous les $2 \mathrm{~mm}$ afin de dépister les tumeurs infracentimétriques. Une amygdalectomie homolatérale à l'adénopathie métastatique offre un taux de détection du primitif qui varie entre $18 \%$ et $44,6 \%$ [61,64,65]. L'écart de rentabilité diagnostique entre les études est expliqué par les différences de recours aux techniques d'imagerie dans le bilan pré opératoire (TDM et TEP-TDM sont de réalisation variable). La rentabilité diagnostique de l'amygdalectomie palatine est supérieure aux biopsies profondes d'amygdales palatines $(29,5 \%$ versus 3,2\% ; p=0,0002) [66]. Une technique récente consiste à réséquer le tissu lymphoépithélial de base de langue par voie transorale, sous assistance robotique ou par chirurgie laser. Une revue de la littérature a montré un rendement diagnostique de 80\% dans l'identification des primitifs lorsqu'une amygdalectomie palatine était associée à une amygdalectomie linguale, par voie transo- 
rale. Les primitifs étaient localisés dans l'amygdale linguale dans 56\% des cas [8]. L'intérêt d'une amygdalectomie palatine bilatérale est discuté. Deux études rapportent un taux de détection du primitif respectivement de $10 \%$ (4/41) [67] et 23\% (5/22) [68] dans l'amygdale palatine controlatérale. L'amygdalectomie palatine bilatérale peut présenter d'autres intérêts : la morbidité est faible ; elle permet de limiter les questionnements des cliniciens devant une fixation amygdalienne controlatérale lors d'une TEP-TDM de suivi ; et des cas de primitifs synchrones découverts de façon fortuite ont été rapportés [69].

\section{Biopsie ganglionnaire et évidemment ganglionnaire}

Une adénopathie suspecte de néoplasie, ou un aspect de kyste branchial bénin à l'imagerie doivent faire l'objet d'une vérification histologique. Une exérèse de toute la masse est réalisée, sous anesthésie générale si les conditions le permettent afin d'y associer une analyse extemporanée et un évidemment ganglionnaire dans le même temps opératoire en cas de métastase de carcinome épidermoïde ou de tumeur nécessitant une chirurgie ganglionnaire [30]. Pour les stades avancés inopérables un traitement par radio chimiothérapie peut être proposé [70].

\section{Classification TNM des CAPI de type épidermoïde}

La mise à jour de la classification TNM de l'AJCC en 2017 [13] inclut les carcinomes épidermoïdes de primitif inconnu de la tête et du cou, avec trois entités. Les formes associées au papillomavirus sont à considérer comme originaires de l'oropharynx, celles associées à l'EBV sont à considérer comme originaire du nasopharynx, et les p16- EBV- ne permettent pas d'être assignées à un site muqueux spécifique des VADS (Tableaux $1 ; 2 ; 3$ ).

L'association au statut p16+ doit être interprétée avec prudence en raison des diagnostics différentiels d'un primitif oropharyngé comme nous l'avons vu précédemment.

2.3 Prise en charge thérapeutique des CAPI de la tête et du cou

Principes généraux devant un adénocarcinome, un carcinome neuroendocrine et un carcinome indifférencié de primitif inconnu

Un évidemment ganglionnaire est généralement réalisé suite au diagnostic de carcinome en analyse extemporanée, en l'absence de métastase infraclaviculaire, lorsque l'adénopathie est extirpable.

Pour un adénocarcinome dont l'origine salivaire est suspectée (niveaux ganglionnaires I,II,III), la problématique réside dans le fait qu'il s'agit d'une tumeur de haut grade du fait des métastases ganglionnaires et peu sensible à la radiothérapie. 
L'évidement ganglionnaire réalisé dans un premier temps va permettre de rechercher un primaire dans les glandes sous maxillaire et sub linguale. Une panendoscopie doit être réalisée dans le même temps afin de rechercher une lésion dans les glandes salivaires accessoires. Si le primitif n'est pas retrouvé, il est alors raisonnable de proposer une parotidectomie dans un second temps[70]. Une radiothérapie adjuvante postopératoire est délivrée selon les critères histologiques.

Pour les carcinomes indifférenciés dont l'origine n'est pas thyroïdienne, le statut EBV détermine la conduite à tenir. Les EBV+ sont à considérer comme originaire du nasopharynx (Tableau 2) et relèvent d'un traitement par radio-chimiothérapie. Les formes EBV-sont à traiter de la même façon que les épidermoïdes. Les carcinomes neuroendocrines dont l'analyse immunohistochimique est évocatrice d'un carcinome de Merkel doivent bénéficier d'un évidemment ganglionnaire associé à une radiothérapie selon les critères histologiques, ainsi que d'un suivi dermatologique.

Traitement des carcinomes épidermö̈des de primitif inconnu : chirurgie

La séquence diagnostique dans la prise en charge d'un CAPI de type épidermoïde opérable inclut un évidement ganglionnaire des niveaux I à IV. La chirurgie peut être envisagée comme une modalité thérapeutique ganglionnaire unique pour les carcinomes épidermoïdes avec une adénopathie N1 (ancienne classification TNM $7^{e}$ édition AJCC) sans rupture capsulaire macroscopique, avec ou sans association à l'HPV, car la radiothérapie adjuvante ne semble pas apporter de bénéfice sur le contrôle local et la survie globale [71,72]. La découverte du primitif au cours d'une chirurgie transorale à visée diagnostique (amygdalectomie palatine et/ou linguale) classe la tumeur T1, mais n'est pas suffisante pour obtenir des marges oncologiques. Pour une prise en charge uniquement chirurgicale, il est nécessaire de compléter la résection par des marges musculaires supplémentaires (pharyngectomie emportant le constricteur moyen du pharynx pour l'amygdale palatine et le muscle lingual pour l'amygdale linguale). La réalisation de ces marges d'emblée au cours de la chirurgie à visée diagnostique augmente cependant la morbidité et le risque de complication chirurgicale. C'est seulement avec des marges chirurgicales satisfaisantes et en l'absence de caractéristiques histologiques péjoratives qu'une radiothérapie complémentaire n'est pas indiquée [70].

\section{Traitement des carcinomes épidermö̈des de primitif inconnu : radiothérapie}

La radiothérapie conformationnelle avec modulation d'intensité (RCMI) est actuellement la technique d'irradiation de choix $[68,73,74]$.

En traitement adjuvant dans les stades précoces, la série de Iganej [75] retrouve un taux de contrôle local pour les pN1 et pN2a sans rupture capsulaire (TNM $7^{\mathrm{e}}$ édition AJCC) de 81\% (13/16) dans le groupe traité par chirurgie seule, versus 89\% (8/9) dans le groupe ayant bénéficié de chirurgie et radiothérapie (p=0,94). Pour les stades N2b, N2c, N3 (TNM 7 édition AJCC) et les adénopathies en rupture capsulaire, une radiothérapie adjuvante semble supérieure à une chirurgie seule ou une radiothérapie seule sur la survie globale et 
spécifique [76]. Aucune de ces études n'a pris en compte le statut HPV. L'absence de connaissance du site tumoral ne permet pas de définir l'indication d'une irradiation cervicale uni ou bilatérale de façon précise en cas de métastase unilatérale. Ligey et al. [77] n’ont pas retrouvé de différence sur la survie globale à 5 ans et le contrôle locorégional après une irradiation unilatérale en comparaison à une irradiation bilatérale sur une série de 95 patients. Une étude suédoise de Hemminki et al. [78] suggère un meilleur contrôle loco régional après une radiothérapie bilatérale par rapport à une irradiation homolatérale. Ce bénéfice sur le contrôle locorégional ne se traduit pas en avantage de survie, et s'explique par un traitement de rattrapage radiochirurgical efficace chez les ayant bénéficié d'une irradiation ganglionnaire unilatérale. À partir de ces données sur séries rétrospectives, une indication d'irradiation ganglionnaire unilatérale semble suffisante dans un premier temps en l'absence d'atteinte ganglionnaire bilatérale, de grade histologique favorable et de négativité EBV [79]. L'irradiation des muqueuses réduit à la fois l'émergence ultérieure de la tumeur primitive qui est estimée à 20\% [29] et la récidive loco régionale, mais ne semble pas avoir d'impact sur la survie [80,81]. Il existe plusieurs protocoles, avec soit des irradiations étendues du nasopharynx au larynx ou soit des irradiations plus focalisées sur l'oropharynx ou le nasopharynx. La supériorité de l'une ou l'autre stratégie n'est pas démontrée faute d'inclusion dans les essais thérapeutiques. Les séquelles postradiques sont plus importantes pour les irradiations étendues. Actuellement, il n'existe aucune recommandation sur une irradiation sélective des sites muqueux en cas d'association à l'HPV ou à l'EBV [13].

\section{Traitement des carcinomes épidermoïdes de primitif inconnu : chimiothérapie}

Aucune étude randomisée chez les patients présentant des CAPI cervicaux n'a démontré l'intérêt de la chimiothérapie. Les indications sont basées sur des résultats de prise en charge de carcinomes épidermoïdes de primitif connu de la tête et du cou. La présence d'une rupture capsulaire ganglionnaire et de marges de résections tumorales positives sont les deux éléments histologiques péjoratifs pour lesquels la chimiothérapie associée à la radiothérapie en post opératoire a permis une amélioration de la survie globale, de la survie sans récidive et du contrôle loco régional [82].

\section{Pronostic des carcinomes épidermö̈des de primitif inconnu}

Une série récente nord-Américaine portant sur une cohorte de 978 patients traités entre 2010 et 2013 a étudié les facteurs pronostiques des CAPI. La survie à 3 ans des CAPI associés à l'HPV retrouvés chez 746 patients était de 94,8\% comparativement aux patients HPV négatifs dont la survie était de 80,3\% (n=232). Aucune différence de survie n'a été mise en évidence selon la modalité thérapeutique employée pour les CAPI associés à l'HPV. Un traitement par radiothérapie seule pour les patients HPV négatifs cN2/N3 était associé à une moins bonne survie comparativement à un traitement multimodal par radio-chimiothérapie ou chirurgie avec radio-chimiothérapie[83]. 


\section{Conclusion}

Les carcinomes de primitif inconnu de la tête et du cou sont des tumeurs rares dont la prise en charge a récemment évolué grâce à la mise en évidence pour certains d'une association à l'HPV ou à l'EBV. Ces associations impactent la présentation clinique. Dans l'avenir, la probable réalisation de nouvelles études permettra sans doute de proposer une "désescalade thérapeutique" et un traitement personnalisé offrant une meilleure qualité de vie. Cependant, il convient de rester prudent et de ne pas inclure dans cette désescalade les CAPI sans association à l'HPV au risque de compromettre les résultats carcinologiques.

\section{Déclaration de lien d'intérêts}

Les auteurs déclarent ne pas avoir de conflits d'intérêts en rapport avec la publication de cet article. 


\section{Références}

[1] Didolkar MS, Fanous N, Elias EG, Moore RH. Metastatic carcinomas from occult primary tumors. A study of 254 patients. Ann Surg 1977;186:625-30.

[2] Waltonen JD, Ozer E, Hall NC, Schuller DE, Agrawal A. Metastatic carcinoma of the neck of unknown primary origin: evolution and efficacy of the modern workup. Arch Otolaryngol Head Neck Surg 2009;135:1024-9. doi:10.1001/archoto.2009.145.

[3] Strojan P, Ferlito A, Medina JE, Woolgar JA, Rinaldo A, Robbins KT, et al. Contemporary management of lymph node metastases from an unknown primary to the neck: I. A review of diagnostic approaches. Head Neck 2013;35:123-32. doi:10.1002/hed.21898.

[4] Issing WJ, Taleban B, Tauber S. Diagnosis and management of carcinoma of unknown primary in the head and neck. Eur Arch Oto-Rhino-Laryngol Off J Eur Fed Oto-Rhino-Laryngol Soc EUFOS Affil Ger Soc Oto-Rhino-Laryngol - Head Neck Surg 2003;260:436-43. doi:10.1007/s00405-003-0585-z.

[5] Nguyen C, Shenouda G, Black MJ, Vuong T, Donath D, Yassa M. Metastatic squamous cell carcinoma to cervical lymph nodes from unknown primary mucosal sites. Head Neck 1994;16:58-63.

[6] Keller LM, Galloway TJ, Holdbrook T, Ruth K, Yang D, Dubyk C, et al. p16 Status, Pathologic and Clinical Characteristics, Biomolecular Signature, and Long Term Outcomes in Unknown Primary Carcinomas of the Head and Neck. Head Neck 2014;36:1677-84. doi:10.1002/hed.23514.

[7] Motz K, Qualliotine JR, Rettig E, Richmon JD, Eisele DW, Fakhry C. Changes in Unknown Primary Squamous Cell Carcinoma of the Head and Neck at Initial Presentation in the Era of Human Papillomavirus. JAMA Otolaryngol-- Head Neck Surg 2016;142:223-8. doi:10.1001/jamaoto.2015.3228.

[8] Fu TS, Foreman A, Goldstein DP, de Almeida JR. The role of transoral robotic surgery, transoral laser microsurgery, and lingual tonsillectomy in the identification of head and neck squamous cell carcinoma of unknown primary origin: a systematic review. J Otolaryngol - Head Neck Surg 2016;45. doi:10.1186/s40463-016-0142-6.

[9] Lacau St Guily J, Rousseau A, Baujat B, Périé S, Schultz P, Barry B, et al. Oropharyngeal cancer prognosis by tumour HPV status in France: The multicentric Papillophar study. Oral Oncol 2017;67:29-36. doi:10.1016/j.oraloncology.2017.01.012.

[10] Perry ME. The specialised structure of crypt epithelium in the human palatine tonsil and its functional significance. J Anat 1994;185 ( Pt 1):111-27.

[11] Black CC, Ogomo C. Does pTis exist in HPV-driven tonsillar carcinomas? An ultrastructural review and examination of two cases. Ultrastruct Pathol 2017;41:55-61. doi:10.1080/01913123.2016.1258020.

[12] Update From the 4th Edition of the World Health Organization Classification of Head and Neck Tumours: Nasopharynx. PubMed J n.d. https://ncbi.nlm.nih.gov/labs/articles/28247232/ (accessed November 28, 2017).

[13] Lydiatt WM, Patel SG, O'Sullivan B, Brandwein MS, Ridge JA, Migliacci JC, et al. Head and Neck cancers-major changes in the American Joint Committee on cancer eighth edition cancer staging manual. CA Cancer J Clin 2017;67:122-37. doi:10.3322/caac.21389.

[14] Lewis JS, Beadle B, Bishop JA, Chernock RD, Colasacco C, Lacchetti C, et al. Human Papillomavirus Testing in Head and Neck Carcinomas: Guideline From the College of American Pathologists. Arch Pathol Lab Med 2017. doi:10.5858/arpa.2017-0286-CP.

[15] McDowell LJ, Young RJ, Johnston ML, Tan T-J, Kleid S, Liu CS, et al. p16-positive lymph node metastases from cutaneous head and neck squamous cell carcinoma: No association with high-risk human papillomavirus or prognosis and implications for the workup of the unknown primary. Cancer 2016;122:1201-8. doi:10.1002/cncr.29901.

[16] Beadle BM, William WN, McLemore MS, Sturgis EM, Williams MD. p16 expression in cutaneous squamous carcinomas with neck metastases: a potential pitfall in identifying unknown primaries of the head and neck. Head Neck 2013;35:1527-33. doi:10.1002/hed.23188.

[17] Maxwell JH, Kumar B, Feng FY, McHugh JB, Cordell KG, Eisbruch A, et al. HPV-positive/p16positive/EBV-negative nasopharyngeal carcinoma in white North Americans. Head Neck 2010;32:562-7. doi:10.1002/hed.21216.

[18] Ndiaye C, Mena M, Alemany L, Arbyn M, Castellsagué X, Laporte L, et al. HPV DNA, E6/E7 mRNA, and p16INK4a detection in head and neck cancers: a systematic review and meta-analysis. Lancet 
Oncol 2014;15:1319-31. doi:10.1016/S1470-2045(14)70471-1.

[19] Ghosh L, Dahut W, Kakar S, Posadas EM, Torres CG, Cancel-Santiago R, et al. Management of patients with metastatic cancer of unknown primary. Curr Probl Surg 2005;42:12-66.

doi:10.1067/j.cpsurg.2004.10.002.

[20] Garrel R, Tripodi C, Cartier C, Makeieff M, Crampette L, Guerrier B. Cervical lymphadenopathies signaling thyroid microcarcinoma. Case study and review of the literature. Eur Ann Otorhinolaryngol Head Neck Dis 2011;128:115-9. doi:10.1016/j.anorl.2010.11.007.

[21] Grani G, Fumarola A. Thyroglobulin in lymph node fine-needle aspiration washout: a systematic review and meta-analysis of diagnostic accuracy. J Clin Endocrinol Metab 2014;99:1970-82.

doi:10.1210/jc.2014-1098.

[22] Costante G, Filetti S. Diagnosis: Thyroglobulin in fine-needle aspirates-a clue to metastasis? Nat Rev Endocrinol 2009;5:249-50. doi:10.1038/nrendo.2009.55.

[23] Karanikas G, Moameni A, Poetzi C, Zettinig G, Kaserer K, Bieglmayer C, et al. Frequency and relevance of elevated calcitonin levels in patients with neoplastic and nonneoplastic thyroid disease and in healthy subjects. J Clin Endocrinol Metab 2004;89:515-9. doi:10.1210/jc.2003-030709.

[24] Vierhapper H, Niederle B, Bieglmayer C, Kaserer K, Baumgartner-Parzer S. Early diagnosis and curative therapy of medullary thyroid carcinoma by routine measurement of serum calcitonin in patients with thyroid disorders. Thyroid Off J Am Thyroid Assoc 2005;15:1267-72. doi:10.1089/thy.2005.15.1267. [25] Bugat R, Sor GDT. Standards, Options et Recommandations 2002 pour la prise en charge des patients atteints de carcinomes de site primitif inconnu (rapport abrégé). Bull Cancer (Paris) 2002;89:869-75. [26] Wong HH, Wang J. Merkel cell carcinoma. Arch Pathol Lab Med 2010;134:1711-6. doi:10.1043/2009-0165-RSR2.1.

[27] Trosman S, Chute D, Wood B, Lamarre E. Unknown primary mucoepidermoid carcinoma: diagnosis and treatment. Head Neck 2015;37:E22-25. doi:10.1002/hed.23766.

[28] López F, Rodrigo JP, Silver CE, Haigentz M, Bishop JA, Strojan P, et al. Cervical lymph node metastases from remote primary tumor sites. Head Neck 2016;38:E2374-85. doi:10.1002/hed.24344.

[29] Grau C, Johansen LV, Jakobsen J, Geertsen P, Andersen E, Jensen BB. Cervical lymph node metastases from unknown primary tumours. Results from a national survey by the Danish Society for Head and Neck Oncology. Radiother Oncol J Eur Soc Ther Radiol Oncol 2000;55:121-9.

[30] SFORL. Adénopathies cervicales chroniques de l'adulte- Recommandation pour la pratique clinique n.d.

[31] SFORL. Adénopathies cervicales d'allure kystique de l'adulte et de l'enfant- Recommandation pour la pratique clinique n.d.

[32] Granström G, Edström S. The relationship between cervical cysts and tonsillar carcinoma in adults. J Oral Maxillofac Surg Off J Am Assoc Oral Maxillofac Surg 1989;47:16-20.

[33] Bradley PT, Bradley PJ. Branchial cleft cyst carcinoma: fact or fiction? Curr Opin Otolaryngol Head Neck Surg 2013;21:118-23. doi:10.1097/MOO.0b013e32835cebde.

[34] Goldenberg D, Begum S, Westra WH, Khan Z, Sciubba J, Pai SI, et al. Cystic lymph node metastasis in patients with head and neck cancer: An HPV-associated phenomenon. Head Neck 2008;30:898-903. doi:10.1002/hed.20796.

[35] Pietarinen-Runtti P, Apajalahti S, Robinson S, Passador-Santos F, Leivo I, Mäkitie AA. Cystic neck lesions: clinical, radiological and differential diagnostic considerations. Acta Otolaryngol (Stockh) 2010;130:300-4. doi:10.3109/00016480903127450.

[36] Regauer S, Mannweiler S, Anderhuber W, Gotschuli A, Berghold A, Schachenreiter J, et al. Cystic lymph node metastases of squamous cell carcinoma of Waldeyer's ring origin. Br J Cancer 1999;79:143742. doi:10.1038/sj.bjc.6690229.

[37] Verma K, Mandal S, Kapila K. Cystic change in lymph nodes with metastatic squamous cell carcinoma. Acta Cytol 1995;39:478-80.

[38] Werner JA, Dünne AA, Myers JN. Functional anatomy of the lymphatic drainage system of the upper aerodigestive tract and its role in metastasis of squamous cell carcinoma. Head Neck 2003;25:322-32. doi:10.1002/hed.10257.

[39] Yeo JCL, Lim SY, Hilmi OJ, MacKenzie K. An analysis of non-head and neck primaries presenting to the neck lump clinic: our experience in two thousand nine hundred and six new patients. Clin Otolaryngol Off J ENT-UK Off J Neth Soc Oto-Rhino-Laryngol Cervico-Facial Surg 2013;38:429-32.

doi:10.1111/coa.12151. 
[40] Lodder W 1., Sewnaik A, Den Bakker M a., Meeuwis C a., Kerrebijn J d. f. Selective neck dissection for $\mathrm{N} 0$ and $\mathrm{N} 1$ oral cavity and oropharyngeal cancer: are skip metastases a real danger? Clin Otolaryngol 2008;33:450-7. doi:10.1111/j.1749-4486.2008.01781.x.

[41] Monnet O, Cohen F, Lecorroller T, Vidal V, Jacquier A, Gaubert JY, et al. [Cervical lymph nodes]. J Radiol 2008;89:1020-36.

[42] Rumboldt Z, Gordon L, Gordon L, Bonsall R, Ackermann S. Imaging in head and neck cancer. Curr Treat Options Oncol 2006;7:23-34.

[43] Ng SH, Chang TC, Ko SF, Yen PS, Wan YL, Tang LM, et al. Nasopharyngeal carcinoma: MRI and CT assessment. Neuroradiology 1997;39:741-6.

[44] Hermans R. Imaging in cervical nodal metastases of unknown primary. Cancer Imaging Off Publ Int Cancer Imaging Soc 2011;11 Spec No A:S9-14. doi:10.1102/1470-7330.2011.9004.

[45] Layfield LJ. Fine-needle aspiration in the diagnosis of head and neck lesions: a review and discussion of problems in differential diagnosis. Diagn Cytopathol 2007;35:798-805. doi:10.1002/dc.20769.

[46] Pfeiffer J, Kayser L, Ridder GJ. Minimal-invasive core needle biopsy of head and neck malignancies: clinical evaluation for radiation oncology. Radiother Oncol J Eur Soc Ther Radiol Oncol 2009;90:2027. doi:10.1016/j.radonc.2008.10.018.

[47] Flezar MS, Kirbis IS, Popović KS, Strojan P. Radiosensitivity of squamous cell carcinoma metastases to the neck assessed by immunocytochemical profiling of fine-needle aspiration biopsy cell specimens: a pilot study. Radiother Oncol J Eur Soc Ther Radiol Oncol 2009;93:575-80.

doi:10.1016/j.radonc.2009.09.007.

[48] Gourin CG, Johnson JT. Incidence of unsuspected metastases in lateral cervical cysts. The Laryngoscope 2000;110:1637-41. doi:10.1097/00005537-200010000-00012.

[49] Üstün M, Risberg B, Davidson B, Berner A. Cystic change in metastatic lymph nodes: A common diagnostic pitfall in fine-needle aspiration cytology. Diagn Cytopathol 2002;27:387-92.

doi:10.1002/dc.10201.

[50] Shah KSV, Ethunandan M. Tumour seeding after fine-needle aspiration and core biopsy of the head and neck--a systematic review. Br J Oral Maxillofac Surg 2016;54:260-5. doi:10.1016/j.bjoms.2016.01.004. [51] McGuirt WF, McCabe BF. Significance of node biopsy before definitive treatment of cervical metastatic carcinoma. The Laryngoscope 1978;88:594-7.

[52] Ellis ER, Mendenhall WM, Rao PV, McCarty PJ, Parsons JT, Stringer SP, et al. Incisional or excisional neck-node biopsy before definitive radiotherapy, alone or followed by neck dissection. Head Neck 1991;13:177-83.

[53] Robbins KT, Cole R, Marvel J, Fields R, Wolf P, Goepfert H. The violated neck: cervical node biopsy prior to definitive treatment. Otolaryngol--Head Neck Surg Off J Am Acad Otolaryngol-Head Neck Surg 1986;94:605-10. doi:10.1177/019459988609400513.

[54] Rusthoven KE, Koshy M, Paulino AC. The role of fluorodeoxyglucose positron emission tomography in cervical lymph node metastases from an unknown primary tumor. Cancer 2004;101:2641-9. doi:10.1002/cncr.20687.

[55] Boellaard R, Delgado-Bolton R, Oyen WJG, Giammarile F, Tatsch K, Eschner W, et al. FDG PET/CT: EANM procedure guidelines for tumour imaging: version 2.0. Eur J Nucl Med Mol Imaging 2015;42:328-54. doi:10.1007/s00259-014-2961-x.

[56] Miller FR, Hussey D, Beeram M, Eng T, McGuff HS, Otto RA. Positron emission tomography in the management of unknown primary head and neck carcinoma. Arch Otolaryngol Head Neck Surg 2005;131:626-9. doi:10.1001/archotol.131.7.626.

[57] de Bree R. The real additional value of FDG-PET in detecting the occult primary tumour in patients with cervical lymph node metastases of unknown primary tumour. Eur Arch Oto-Rhino-Laryngol Off J Eur Fed Oto-Rhino-Laryngol Soc EUFOS Affil Ger Soc Oto-Rhino-Laryngol - Head Neck Surg 2010;267:1653-5. doi:10.1007/s00405-010-1372-2.

[58] Gutzeit A, Antoch G, Kühl H, Egelhof T, Fischer M, Hauth E, et al. Unknown primary tumors: detection with dual-modality PET/CT--initial experience. Radiology 2005;234:227-34.

doi:10.1148/radiol.2341031554.

[59] Padovani D, Aimoni C, Zucchetta P, Paluzzi A, Pastore A. 18-FDG PET in the diagnosis of laterocervical metastases from occult carcinoma. Eur Arch Oto-Rhino-Laryngol Off J Eur Fed Oto-RhinoLaryngol Soc EUFOS Affil Ger Soc Oto-Rhino-Laryngol - Head Neck Surg 2009;266:267-71. doi:10.1007/s00405-008-0733-6. 
[60] Roh J-L, Kim JS, Lee JH, Cho K-J, Choi S-H, Nam SY, et al. Utility of combined (18)Ffluorodeoxyglucose-positron emission tomography and computed tomography in patients with cervical metastases from unknown primary tumors. Oral Oncol 2009;45:218-24.

doi:10.1016/j.oraloncology.2008.05.010.

[61] Cianchetti M, Mancuso AA, Amdur RJ, Werning JW, Kirwan J, Morris CG, et al. Diagnostic evaluation of squamous cell carcinoma metastatic to cervical lymph nodes from an unknown head and neck primary site. The Laryngoscope 2009;119:2348-54. doi:10.1002/lary.20638.

[62] Hosni A, Dixon PR, Rishi A, Au M, Xu W, Song Y, et al. Radiotherapy Characteristics and Outcomes for Head and Neck Carcinoma of Unknown Primary vs T1 Base-of-Tongue Carcinoma. JAMA Otolaryngol-- Head Neck Surg 2016. doi:10.1001/jamaoto.2016.3083.

[63] Patel SA, Parvathaneni A, Parvathaneni U, Houlton JJ, Karni RJ, Liao JJ, et al. Post-operative therapy following transoral robotic surgery for unknown primary cancers of the head and neck. Oral Oncol 2017;72:150-6. doi:10.1016/j.oraloncology.2017.07.019.

[64] Haas I, Hoffmann TK, Engers R, Ganzer U. Diagnostic strategies in cervical carcinoma of an unknown primary (CUP). Eur Arch Oto-Rhino-Laryngol Off J Eur Fed Oto-Rhino-Laryngol Soc EUFOS Affil Ger Soc Oto-Rhino-Laryngol - Head Neck Surg 2002;259:325-33. doi:10.1007/s00405-002-0470-1.

[65] Lapeyre M, Malissard L, Peiffert D, Hoffstetter S, Toussaint B, Renier S, et al. Cervical lymph node metastasis from an unknown primary: is a tonsillectomy necessary? Int J Radiat Oncol Biol Phys 1997;39:291-6.

[66] Waltonen JD, Ozer E, Schuller DE, Agrawal A. Tonsillectomy vs. deep tonsil biopsies in detecting occult tonsil tumors. The Laryngoscope 2009;119:102-6. doi:10.1002/lary.20017.

[67] Koch WM, Bhatti N, Williams MF, Eisele DW. Oncologic rationale for bilateral tonsillectomy in head and neck squamous cell carcinoma of unknown primary source. Otolaryngol--Head Neck Surg Off J Am Acad Otolaryngol-Head Neck Surg 2001;124:331-3. doi:10.1067/mhn.2001.114309.

[68] Kothari P, Randhawa PS, Farrell R. Role of tonsillectomy in the search for a squamous cell carcinoma from an unknown primary in the head and neck. Br J Oral Maxillofac Surg 2008;46:283-7. doi:10.1016/j.bjoms.2007.11.017.

[69] Rasband-Lindquist A, Shnayder Y, O’Neil M. Synchronous bilateral tonsillar squamous cell carcinoma related to human papillomavirus: Two case reports and a brief review of the literature. Ear Nose Throat J 2016;95:E30-34.

[70] National Comprehensive Cancer Network. NCCN Guidelines Version 2.2017 Head and Neck Cancers n.d. https://www.nccn.org/professionals/physician_gls/pdf/head-and-neck.pdf.

[71] Strojan P, Ferlito A, Langendijk JA, Corry J, Woolgar JA, Rinaldo A, et al. Contemporary management of lymph node metastases from an unknown primary to the neck: II. a review of therapeutic options. Head Neck 2013;35:286-93. doi:10.1002/hed.21899.

[72] Strojan P, Ferlito A, Langendijk JA, Silver CE. Indications for radiotherapy after neck dissection. Head Neck 2012;34:113-9. doi:10.1002/hed.21599.

[73] Frank SJ, Rosenthal DI, Petsuksiri J, Ang KK, Morrison WH, Weber RS, et al. Intensity-modulated radiotherapy for cervical node squamous cell carcinoma metastases from unknown head-and-neck primary site: M. D. Anderson Cancer Center outcomes and patterns of failure. Int J Radiat Oncol Biol Phys 2010;78:1005-10. doi:10.1016/j.ijrobp.2009.09.006.

[74] Nutting CM, Morden JP, Harrington KJ, Urbano TG, Bhide SA, Clark C, et al. Parotid-sparing intensity modulated versus conventional radiotherapy in head and neck cancer (PARSPORT): a phase 3 multicentre randomised controlled trial. Lancet Oncol 2011;12:127-36. doi:10.1016/S1470-2045(10)70290-4.

[75] Iganej S, Kagan R, Anderson P, Rao A, Tome M, Wang R, et al. Metastatic squamous cell carcinoma of the neck from an unknown primary: Management options and patterns of relapse. Head Neck 2002;24:236-46. doi:10.1002/hed.10017.

[76] Wallace A, Richards GM, Harari PM, Kirwan JM, Morris CG, Katakam H, et al. Head and neck squamous cell carcinoma from an unknown primary site. Am J Otolaryngol 2011;32:286-90. doi:10.1016/j.amjoto.2010.05.004.

[77] Ligey A, Gentil J, Créhange G, Montbarbon X, Pommier P, Peignaux K, et al. Impact of target volumes and radiation technique on loco-regional control and survival for patients with unilateral cervical lymph node metastases from an unknown primary. Radiother Oncol J Eur Soc Ther Radiol Oncol 2009;93:483-7. doi:10.1016/j.radonc.2009.08.027.

[78] Hemminki K, Bevier M, Hemminki A, Sundquist J. Survival in cancer of unknown primary site: 
population-based analysis by site and histology. Ann Oncol Off J Eur Soc Med Oncol 2012;23:1854-63. doi:10.1093/annonc/mdr536.

[79] Troussier I, Barry B, Baglin AC, Leysalle A, Janot F, Baujat B, et al. [Target volumes in cervical lympadenopathies of unknown primary: toward a selective customized approach? On behalf of REFCOR]. Cancer Radiother J Soc Francaise Radiother Oncol 2013;17:686-94. doi:10.1016/j.canrad.2013.07.132.

[80] Colletier PJ, Garden AS, Morrison WH, Goepfert H, Geara F, Ang KK. Postoperative radiation for squamous cell carcinoma metastatic to cervical lymph nodes from an unknown primary site: outcomes and patterns of failure. Head Neck 1998;20:674-81.

[81] Erkal HS, Mendenhall WM, Amdur RJ, Villaret DB, Stringer SP. Squamous cell carcinomas metastatic to cervical lymph nodes from an unknown head-and-neck mucosal site treated with radiation therapy alone or in combination with neck dissection. Int J Radiat Oncol Biol Phys 2001;50:55-63.

[82] Cooper JS, Pajak TF, Forastiere AA, Jacobs J, Campbell BH, Saxman SB, et al. Postoperative concurrent radiotherapy and chemotherapy for high-risk squamous-cell carcinoma of the head and neck. N Engl J Med 2004;350:1937-44. doi:10.1056/NEJMoa032646.

[83] Cheraghlou S, Torabi SJ, Husain ZA, Otremba MD, Osborn HA, Mehra S, et al. HPV status in unknown primary head and neck cancer: Prognosis and treatment outcomes. The Laryngoscope 2018.

doi:10.1002/lary.27475. 
Figure 1. Adénopathie métastatique d'un carcinome épidermoïde non kératinisant HPV induit, coloration hématoxyline éosine, faible grossissement (Pr V. Costes)

L'analyse morphologique montre un ganglion creusé d'une cavité kystique (1) limitée par une prolifération épithéliale non ou peu kératinisée (2), constituée de cellules ovales ou discrètement fusiformes, à limites cytoplasmiques floues, et aux hauts rapports nucléocytoplasmiques.

Figure 2. Algorithme décisionnel pour la recherche du statut HPV et EBER

Figure 3. Algorithme décisionnel devant une adénopathie prévalente

Figure 4. Premiers échelons de drainage lymphatique par sites de la tête et du cou [38] 
Tableau 1. Cancer de l'oropharynx associé à l'HPV de primitif inconnu ; classification TNM 2017 de l’AJCC/ UICC.

\begin{tabular}{|c|c|c|c|}
\hline \multicolumn{4}{|l|}{ Tumeur (T) } \\
\hline T0 & \multicolumn{3}{|c|}{$\begin{array}{l}\text { Pas de primitif identifié mais association au papillomavirus retrouvée dans } \\
\text { une adénopathie }\end{array}$} \\
\hline \multicolumn{4}{|l|}{ Ganglions cervicaux $(\mathbf{N})$} \\
\hline Classification clinique $(\mathrm{cN})$ & \multicolumn{3}{|c|}{ Pour les patients traités sans évidemment ganglionnaire } \\
\hline $\mathrm{cNx}$ & \multicolumn{3}{|c|}{ Le statut ganglionnaire ne peut pas être évalué } \\
\hline $\mathrm{cNO}$ & \multicolumn{3}{|c|}{ Pas d'atteinte ganglionnaire } \\
\hline $\mathrm{cN} 1$ & \multicolumn{3}{|c|}{ Adénopathie(s) unilatérale(s) de moins de $6 \mathrm{~cm}$} \\
\hline $\mathrm{cN} 2$ & \multicolumn{3}{|c|}{ Adénopathie(s) controlatérale(s) ou bilatérale(s) de moins de $6 \mathrm{~cm}$} \\
\hline $\mathrm{cN} 3$ & \multicolumn{3}{|c|}{ Adénopathie(s) de plus de $6 \mathrm{~cm}$} \\
\hline $\begin{array}{l}\text { Classification pathologique } \\
(\mathrm{pN})\end{array}$ & \multicolumn{3}{|c|}{$\begin{array}{l}\text { Pour les patients traités avec évidemment ganglionnaire et analyse histolo- } \\
\text { gique }\end{array}$} \\
\hline $\mathrm{pNx}$ & \multicolumn{3}{|c|}{ Le statut ganglionnaire ne peut pas être évalué } \\
\hline pNO & \multicolumn{3}{|c|}{ Pas d'atteinte ganglionnaire } \\
\hline pN1 & \multicolumn{3}{|c|}{ Métastase(s) dans 4 ganglions lymphatiques ou moins } \\
\hline $\mathrm{pN} 2$ & \multicolumn{3}{|c|}{ Métastases dans plus de 4 ganglions } \\
\hline \multicolumn{4}{|l|}{ Métastase à distance (M) } \\
\hline MX & \multicolumn{3}{|c|}{ Le statut métastatique à distance n'est pas évalué } \\
\hline M0 & \multicolumn{3}{|c|}{ Pas de métastase à distance } \\
\hline M1 & \multicolumn{3}{|c|}{ Métastase(s) à distance } \\
\hline \multicolumn{4}{|l|}{ Stades pronostiques } \\
\hline $\mathrm{T}$ & $\mathrm{N}$ & $\mathrm{M}$ & Stade \\
\hline T0 & N1 & M0 & I \\
\hline T0 & $\mathrm{N} 2$ & M0 & II \\
\hline T0 & N3 & M0 & III \\
\hline T0 & Tous $\mathrm{N}$ & M1 & IV \\
\hline
\end{tabular}

TNM : Tumor, Node, Metastasis ; AJCC : American Joint Committee on Cancer ; UICC : Union for International Cancer Control ; HPV : Human Papillomavirus 
Tableau 2. Cancer du nasopharynx de primitif inconnu ; classification TNM 2017 de l'AJCC/ UICC.

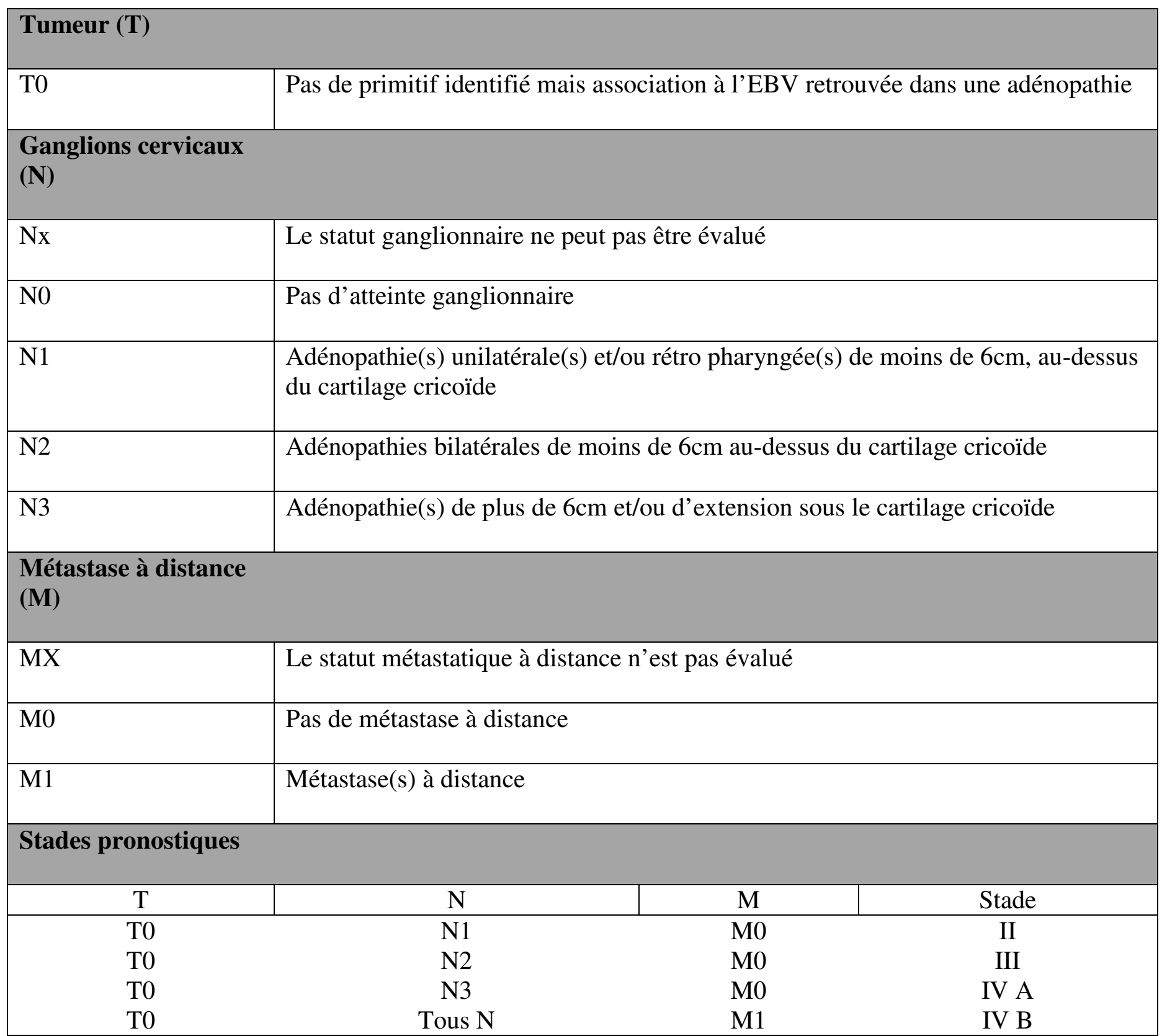

TNM : Tumor, Node, Metastasis ; AJCC : American Joint Committee on Cancer ; UICC : Union for International Cancer Control ; EBV : Epstein-Barr Virus 
Tableau 3. Carcinome épidermoïde de primitif inconnu de la tête et du cou, sans association à l'HPV ni à l'EBV; classification TNM 2017 de l'AJCC/ UICC.

\begin{tabular}{|c|c|}
\hline \multicolumn{2}{|l|}{ Tumeur (T) } \\
\hline T0 & Pas de primitif identifié \\
\hline \multicolumn{2}{|l|}{ Ganglions cervicaux $(\mathbf{N})$} \\
\hline Classification clinique $(\mathrm{cN})$ & Pour les patients traités sans évidemment ganglionnaire \\
\hline $\mathrm{cNx}$ & Le statut ganglionnaire ne peut pas être évalué \\
\hline $\mathrm{cN} 1$ & Métastase ganglionnaire unique, ENEc(-) \\
\hline $\mathrm{cN} 2 \mathrm{a}$ & Métastase ganglionnaire unique homolatérale $>3 \mathrm{~cm}, \leq 6 \mathrm{~cm}$, ENEc $(-)$ \\
\hline $\mathrm{cN} 2 \mathrm{~b}$ & Métastases ganglionnaires multiples homolatérales $\leq 6 \mathrm{~cm}$, ENEc (-) \\
\hline $\mathrm{cN} 2 \mathrm{c}$ & Métastases ganglionnaires bilatérales $\leq 6 \mathrm{~cm}$, ENEc (-) \\
\hline $\mathrm{cN} 3 \mathrm{a}$ & Métastase(s) ganglionnaire(s)>6cm, ENEc (-) \\
\hline $\mathrm{cN} 3 \mathrm{~b}$ & Métastase(s) ganglionnaire(s) ENEc (+) \\
\hline \multicolumn{2}{|c|}{$\begin{array}{l}\text { Une rupture capsulaire clinique (ENEc) est définie par une atteinte de la peau, une fixation aux structures } \\
\text { musculaires ou vasculaires sous-jacentes, des signes cliniques d'atteinte nerveuse (nerf(s) crânien(s), plexus } \\
\text { brachial, nerf phrénique, tronc sympathique) }\end{array}$} \\
\hline Classification pathologique $(\mathrm{pN})$ & $\begin{array}{l}\text { Pour les patients traités avec évidemment ganglionnaire et analyse his- } \\
\text { tologique }\end{array}$ \\
\hline $\mathrm{pNx}$ & Le statut ganglionnaire ne peut pas être évalué \\
\hline pNO & Pas d'atteinte ganglionnaire \\
\hline $\mathrm{pN} 1$ & Métastase ganglionnaire homolatérale $\leq 3 \mathrm{~cm}$ et ENEp (-) \\
\hline $\mathrm{pN} 2 \mathrm{a}$ & $\begin{array}{l}\text { Métastase ganglionnaire unique homolatérale } \leq 3 \mathrm{~cm} \text { et } \operatorname{ENEp(+)} \underline{\text { ou }} \\
\text { Métastase ganglionnaire unique homolatérale }>3 \mathrm{~cm}, \leq 6 \mathrm{~cm}, \operatorname{ENEp~(-)}\end{array}$ \\
\hline $\mathrm{pN} 2 \mathrm{~b}$ & Métastases ganglionnaires homolatérales $\leq 6 \mathrm{~cm}$, ENEp (-) \\
\hline $\mathrm{pN} 2 \mathrm{c}$ & $\begin{array}{l}\text { Métastases ganglionnaires bilatérales ou controlatérales } \leq 6 \mathrm{~cm} \text {, ENEp (- } \\
\text { ) }\end{array}$ \\
\hline $\mathrm{pN} 3 \mathrm{a}$ & Métastase(s) ganglionnaire(s) $>6 \mathrm{~cm}$, ENEp (-) \\
\hline $\mathrm{pN} 3 \mathrm{~b}$ & $\begin{array}{l}\text { Métastase ganglionnaire unique homolatérale }>3 \mathrm{~cm} \text { et ENEp (+) ou } \\
\text { Métastase ganglionnaire unique controlatérale de } 3 \mathrm{~cm} \text { ou moins ENEp } \\
(+) \underline{\text { ou }} \\
\text { Métastases ganglionnaires multiples homolatérales, controlatérales ou } \\
\text { bilatérales avec ENEp (+) sans critère de taille }\end{array}$ \\
\hline
\end{tabular}




\begin{tabular}{|c|c|c|c|}
\hline $\begin{array}{l}\text { Une rupture capsu } \\
\leq 2 \mathrm{~mm} \text { ou majeur } \\
\text { dans la classificati }\end{array}$ & $\begin{array}{l}\text { histologique }(E N E p \\
\text { s extensions }>2 \mathrm{~mm} \text {. }\end{array}$ & $\begin{array}{l}\text { inim } \\
\text { a son }\end{array}$ & $\begin{array}{l}\text { les exte } \\
\text { omme } 1\end{array}$ \\
\hline Métastase à dista & & & \\
\hline MX & Le statut métastatic & st pa & \\
\hline M0 & Pas de métastase à & & \\
\hline M1 & Métastase à distan & & \\
\hline Stades pronostiqu & & & \\
\hline $\mathrm{T}$ & $\mathrm{N}$ & M & Stade \\
\hline T0 & N1 & M0 & III \\
\hline T0 & $\mathrm{N} 2$ & M0 & IVA \\
\hline T0 & N3 & M0 & IVB \\
\hline T0 & Tous N & M1 & IVC \\
\hline
\end{tabular}

TNM : Tumor, Node, Metastasis ; AJCC : American Joint Committee on Cancer ; UICC : Union for International Cancer Control ; HPV : Human Papillomavirus ; EBV : Epstein-Barr Virus ; ENE : ExtraNodal Extension (ganglion en rupture capsulaire) 


\section{Figure 1.}




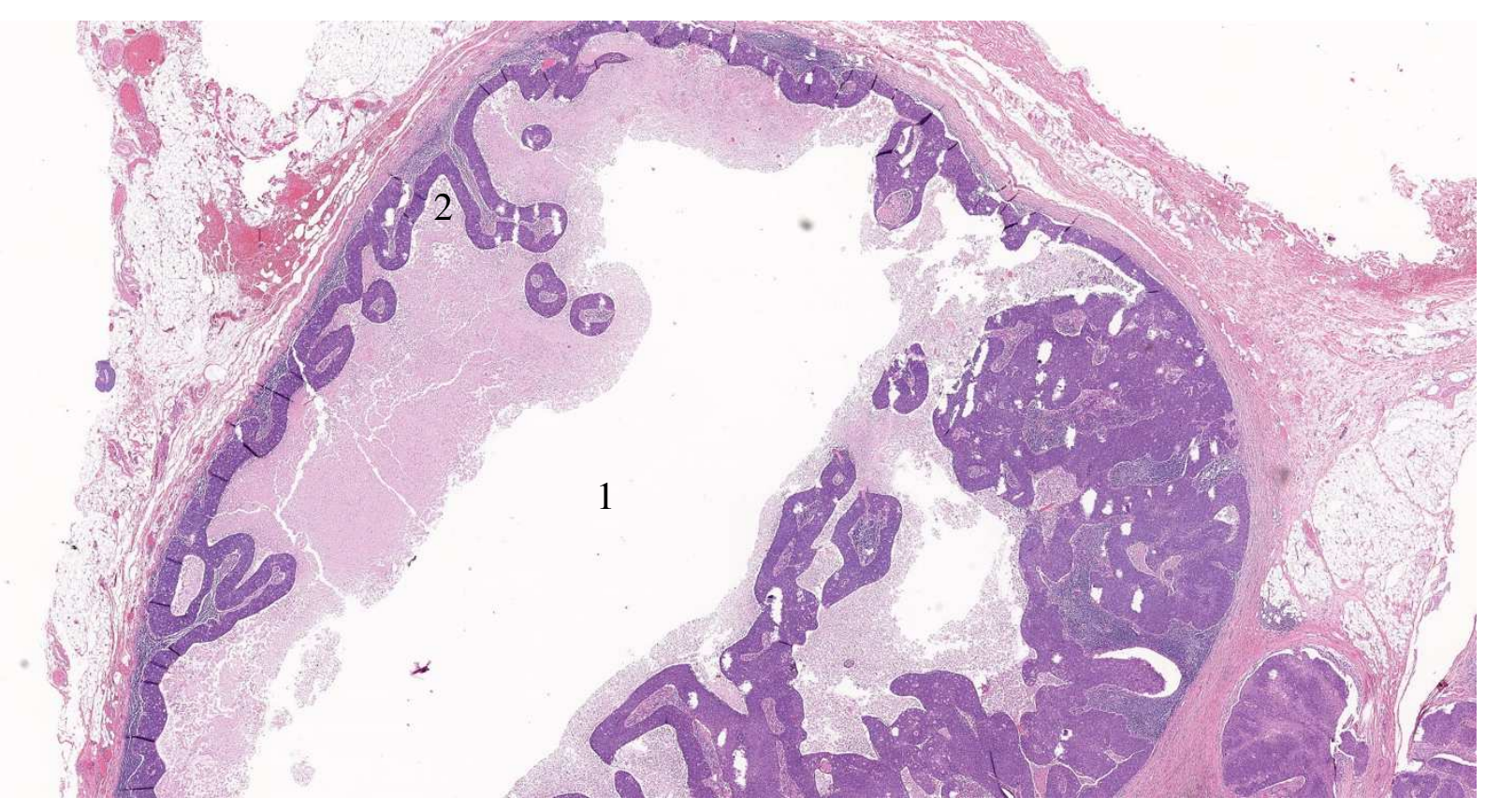


Figure 2.

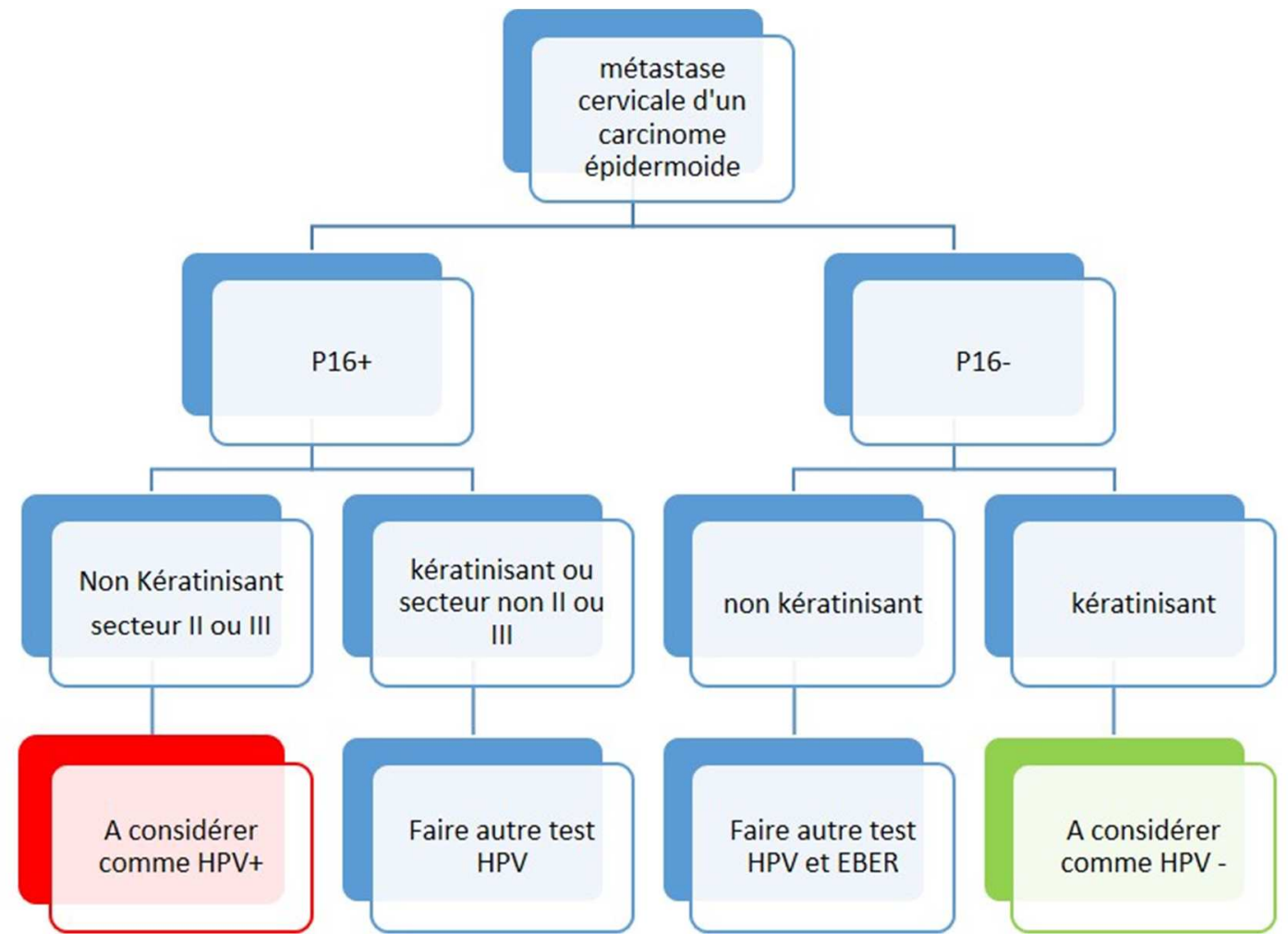




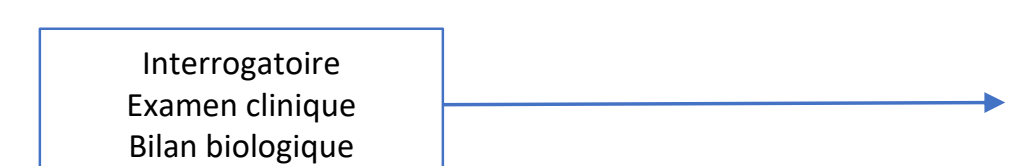

Bilan biologique

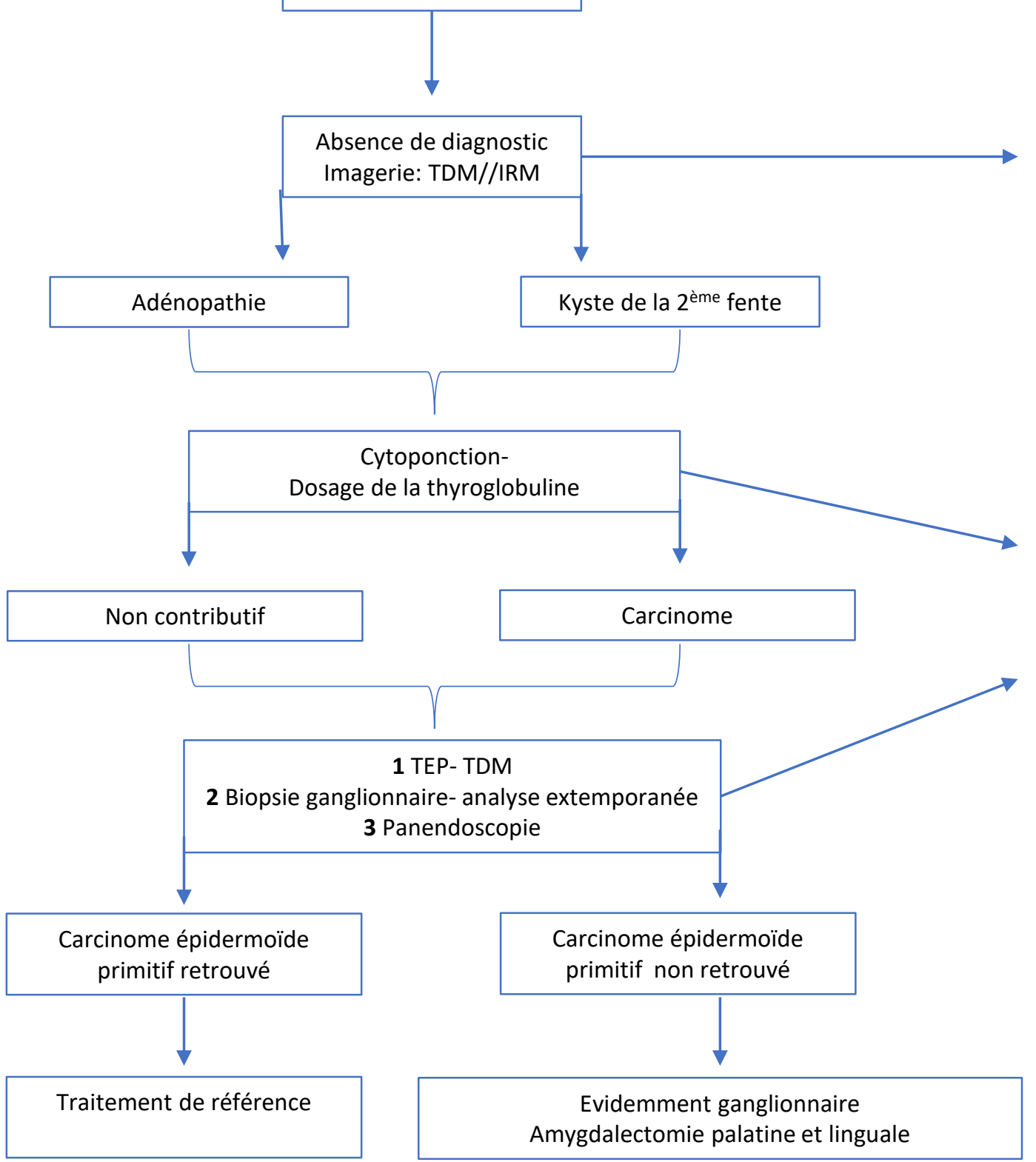

- Primitif cutané/VADS/ Thyroïde

- Origine salivaire

- Origine infectieuse

- Origine inflammatoire

Diagnostics différentiels:
- Paragangliome
- Neurinome
- Lipome
- Etc..

- Primitif thyroïdien (dosage de la thyroglobuline)

- Primitif salivaire

- Histologies non épithéliales (lymphomes, tumeurs germinales,

mélanomes, sarcomes)

Pathologie bénigne*

*une adénopathie kystique est source de faux négatif à la cytoponction, qui peut être faussement rassurante 
TDM: Tomodensitométrie; IRM: imagerie par résonnance magnétique; VADS: voies aéro digestives supérieures; TEP-TDM: tomographie par émission de positon couplée à une tomodensitométrie 
Figure 4.

\section{Zone IIB}

Nasopharynx, Glande parotide, Oropharynx

Zones IIa, III, IV

Cavité orale, Oropharynx, Larynx, Hypopharynx,

Thyroïde

Zones Va, Vb Nasopharynx, Thyroïde

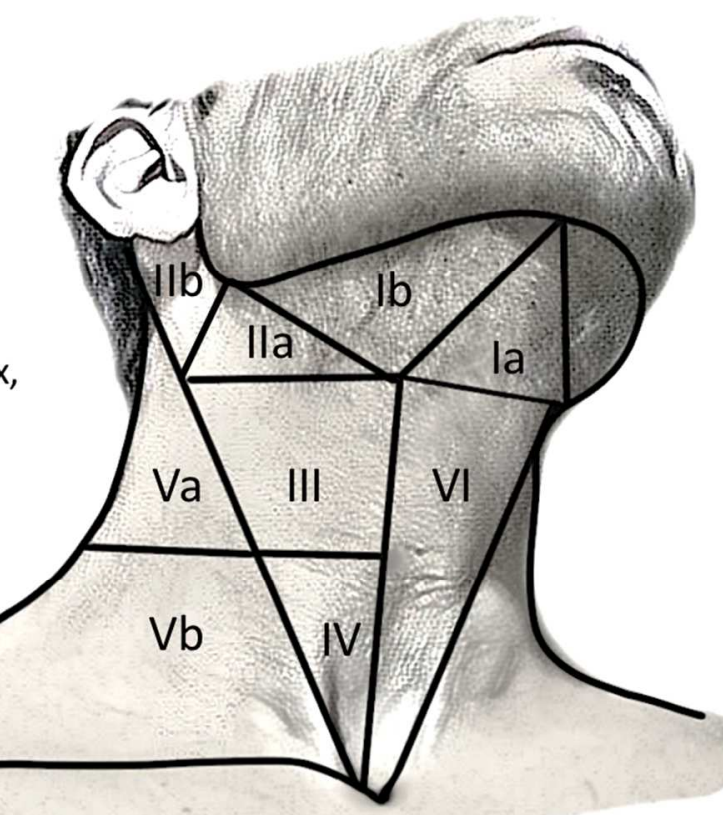

Zones la, lb

Cavité Orale, Lèvre,

Glande sous maxillaire

\section{Zone VI}

Thyroïde, Larynx, Hypopharynx, Oesophage cervical 